Bull. Korean Math. Soc. 52 (2015), No. 3, pp. 987-998

http://dx.doi.org/10.4134/BKMS.2015.52.3.987

\title{
AN INTEGRAL REPRESENTATION, SOME INEQUALITIES, AND COMPLETE MONOTONICITY OF THE BERNOULLI NUMBERS OF THE SECOND KIND
}

\author{
Feng Qi And XiaO-Jing Zhang
}

\begin{abstract}
In the paper, the authors discover an integral representation, some inequalities, and complete monotonicity of the Bernoulli numbers of the second kind.
\end{abstract}

\section{Introduction}

In number theory, the Bernoulli numbers of the second kind $b_{n}$ for $n \in \mathbb{N}_{0}=$ $\mathbb{N} \cup\{0\}$ may be generated by

$$
\frac{x}{\ln (1+x)}=\sum_{n=0}^{\infty} b_{n} x^{n}
$$

where $\mathbb{N}$ denotes the set of positive integers. They are also known as the Cauchy numbers of the first kind (see [5, p. 294]), the Gregory coefficients, or logarithmic numbers. The first few Bernoulli numbers of the second kind $b_{n}$ are

$$
b_{0}=1, \quad b_{1}=\frac{1}{2}, \quad b_{2}=-\frac{1}{12}, \quad b_{3}=\frac{1}{24}, \quad b_{4}=-\frac{19}{720}, \quad b_{5}=\frac{3}{160} .
$$

The first main result of this paper is the following integral representation of $b_{n}$ for $n \in \mathbb{N}$.

Theorem 1. The Bernoulli numbers of the second kind $b_{n}$ may be represented as

$$
b_{n}=(-1)^{n+1} \int_{1}^{\infty} \frac{1}{\left\{[\ln (t-1)]^{2}+\pi^{2}\right\} t^{n}} \mathrm{~d} t, \quad n \in \mathbb{N} .
$$

Received June 24, 2014; Revised January 3, 2015.

2010 Mathematics Subject Classification. Primary 11B68; Secondary 11B83, 26A48, 30E20, 33B99.

Key words and phrases. Bernoulli numbers of the second kind, integral representation, inequality, completely monotonic sequence, Cauchy integral formula. 
Recall from [21, p. 108, Definition 4] that a sequence $\left\{\mu_{n}\right\}_{0<n<\infty}$ is said to be completely monotonic if its elements are non-negative and its successive differences are alternatively non-negative, that is

$$
(-1)^{k} \Delta^{k} \mu_{n} \geq 0, \quad k, n \in \mathbb{N}_{0},
$$

where

$$
\Delta^{k} \mu_{n}=\sum_{m=0}^{k}(-1)^{m}\left(\begin{array}{c}
k \\
m
\end{array}\right) \mu_{n+k-m} .
$$

Recall from $[21$, p. 163, Definition 14a] that a completely monotonic sequence $\left\{a_{n}\right\}_{n \geq 0}$ is minimal if it ceases to be completely monotonic when $a_{0}$ is decreased.

Let $\lambda=\left(\lambda_{1}, \lambda_{2}, \ldots, \lambda_{n}\right) \in \mathbb{R}^{n}$ and $\mu=\left(\mu_{1}, \mu_{2}, \ldots, \mu_{n}\right) \in \mathbb{R}^{n}$. A sequence $\lambda$ is said to be majorized by $\mu$ (in symbols $\lambda \preceq \mu$ ) if

$$
\sum_{\ell=1}^{k} \lambda_{[\ell]} \leq \sum_{\ell=1}^{k} \mu_{[\ell]}, \quad k=1,2, \ldots, n-1 \quad \text { and } \quad \sum_{\ell=1}^{n} \lambda_{\ell}=\sum_{\ell=1}^{n} \mu_{\ell},
$$

where $\lambda_{[1]} \geq \lambda_{[2]} \geq \cdots \geq \lambda_{[n]}$ and $\mu_{[1]} \geq \mu_{[2]} \geq \cdots \geq \mu_{[n]}$ are respectively the components of $\lambda$ and $\mu$ in decreasing order. A sequence $\lambda$ is said to be strictly majorized by $\mu$ (in symbols $\lambda \prec \mu$ ) if $\lambda$ is not a permutation of $\mu$. For example,

$$
\left(\frac{1}{n}, \ldots, \frac{1}{n}\right) \prec\left(\frac{1}{n-1}, \ldots, \frac{1}{n-1}, 0\right) \prec\left(\frac{1}{2}, \frac{1}{2}, 0, \ldots, 0\right) \prec(1,0, \ldots, 0) .
$$

For more information on the theory of majorization and its applications, please refer to monographs $[8,9]$ and closely related references therein.

Based on Theorem 1, the following inequalities and properties of the Bernoulli numbers of the second kind $b_{n}$ are discovered.

Theorem 2. The infinite sequence $\left\{(-1)^{n} b_{n+1}\right\}_{n \geq 0}$ is completely monotonic and minimal.

Theorem 3. Let $m \in \mathbb{N}$ and $a_{k}$ for $1 \leq k \leq m$ be nonnegative integers. Then

$$
\left|\left(a_{k}+a_{j}\right) ! b_{a_{k}+a_{j}+1}\right|_{m} \geq 0
$$

and

$$
\left|(-1)^{a_{k}+a_{j}}\left(a_{k}+a_{j}\right) ! b_{a_{k}+a_{j}+1}\right|_{m} \geq 0,
$$

where $\left|a_{k j}\right|_{m}$ denotes a determinant of order $m$ with elements $a_{k j}$.

Theorem 4. Let $m \in \mathbb{N}$ and let $\lambda$ and $\mu$ be two $m$-tuples of nonnegative numbers such that $\lambda \preceq \mu$. Then

$$
\left|\prod_{\ell=1}^{m} \lambda_{\ell} ! b_{\lambda_{\ell}+1}\right| \leq\left|\prod_{\ell=1}^{m} \mu_{\ell} ! b_{\mu_{\ell}+1}\right| .
$$

Corollary 1. The infinite sequence $\left\{(-1)^{n} n ! b_{n+1}\right\}_{n \geq 0}$ is logarithmically convex. 
INTEGRAL REPRESENTATION AND PROPERTIES OF BERNOULLI NUMBERS 989

\section{Lemmas}

To prove our main results, we need the following two integral representations.

Lemma 1 ([3, p. 2130]). Let $\mathbb{C}$ be the set of complex numbers and let

$$
\ln z=\ln |z|+i \arg z
$$

be the principal branch of the holomorphic extension of $\ln x$ from the open halfline $(0, \infty)$ to the cut plane

$$
\mathcal{A}=\mathbb{C} \backslash(-\infty, 0],
$$

where $-\pi<\arg z<\pi$ and $i=\sqrt{-1}$ is the imaginary unit. The function $\frac{1}{\ln (1+z)}$ for $z \in \mathbb{C} \backslash(-\infty, 0]$ has the integral representation

$$
\frac{1}{\ln (1+z)}=\frac{1}{z}+\int_{1}^{\infty} \frac{1}{[\ln (t-1)]^{2}+\pi^{2}} \frac{\mathrm{d} t}{z+t}
$$

Lemma 2. The function

$$
F(z)= \begin{cases}\frac{z}{(1+z) \ln (1+z)}, & z \in \mathbb{C} \backslash(-\infty,-1] \backslash\{0\} \\ 1, & z=0\end{cases}
$$

has the integral representation

$$
F(z)=\int_{0}^{\infty} \frac{t+1}{t\left[(\ln t)^{2}+\pi^{2}\right]} \frac{\mathrm{d} t}{t+1+z}, \quad z \in \mathbb{C} \backslash(-\infty,-1] .
$$

First proof of Lemma 2. For $z=\varepsilon e^{\theta i}$ with $\theta \in\left[-\frac{\pi}{2}, \frac{\pi}{2}\right]$ and $\varepsilon \in(0,1)$, by standard argument, we have

$$
|z F(z-1)|^{2}=\left|\frac{\varepsilon e^{\theta i}-1}{\ln \left(\varepsilon e^{\theta i}\right)}\right|^{2}=\frac{1-2 \varepsilon \cos \theta+\varepsilon^{2}}{(\ln \varepsilon)^{2}+\theta^{2}} \rightarrow 0
$$

uniformly as $\varepsilon \rightarrow 0^{+}$. Consequently,

$$
\lim _{\varepsilon \rightarrow 0^{+}}[z F(z-1)]=0
$$

uniformly.

For $\theta \in(-\pi, \pi)$ and $z=r e^{\theta i}$, by standard argument, we have

$$
|F(z-1)|=\left|\frac{r e^{\theta i}-1}{r e^{\theta i} \ln \left(r e^{\theta i}\right)}\right|=\sqrt{\frac{1+2 r \cos \theta+r^{2}}{r^{2}\left[(\ln r)^{2}+\theta^{2}\right]}} \rightarrow 0
$$

uniformly as $r \rightarrow \infty$.

For $t \in(0, \infty)$ and $\varepsilon \in(0,1)$, we have

$$
\begin{aligned}
F(-t-1+\varepsilon i) & =\frac{-t-1+\varepsilon i}{(-t+\varepsilon i) \ln (-t+\varepsilon i)} \\
& =\frac{-t-1+\varepsilon i}{(-t+\varepsilon i)[\ln |-t+\varepsilon i|+i \arg (-t+\varepsilon i)]}
\end{aligned}
$$




$$
\begin{aligned}
& =\frac{-t-1+\varepsilon i}{(-t+\varepsilon i)\left[\ln |-t+\varepsilon i|+i\left(\pi-\arctan \frac{\varepsilon}{t}\right)\right]} \\
& \rightarrow \frac{t+1}{t(\ln t+\pi i)} \\
& =\frac{(t+1)(\ln t-\pi i)}{t\left[(\ln t)^{2}+\pi^{2}\right]}
\end{aligned}
$$

as $\varepsilon \rightarrow 0^{+}$. In other words, for $t \in(0, \infty)$,

$$
\lim _{\varepsilon \rightarrow 0^{+}} \Im F(-t-1+\varepsilon i)=-\frac{\pi(t+1)}{t\left[(\ln t)^{2}+\pi^{2}\right]} .
$$

Let $D$ be a bounded domain with piecewise smooth boundary. If $f(z)$ is analytic on $D$ and extendable smoothly to the boundary of $D$, then

$$
f(z)=\frac{1}{2 \pi i} \oint_{\partial D} \frac{f(w)}{w-z} \mathrm{~d} w, \quad z \in D,
$$

which is known as the Cauchy integral formula. See [7, p. 113]. For any fixed point $z_{0}=x_{0}+i y_{0} \in \mathbb{C} \backslash(-\infty, 0]$, choose $\varepsilon$ and $r$ such that

$$
\begin{cases}0<\varepsilon<\left|y_{0}\right| \leq\left|z_{0}\right|<r, & y_{0} \neq 0, \\ 0<\varepsilon<x_{0}=\left|z_{0}\right|<r, & y_{0}=0,\end{cases}
$$

and consider the positively oriented contour $C(\varepsilon, r)$ in $\mathbb{C} \backslash(-\infty,-1]$ consisting of the half circle $z=-1+\varepsilon e^{\theta i}$ for $\theta \in\left[-\frac{\pi}{2}, \frac{\pi}{2}\right]$ and the half lines $z=-1+x \pm \varepsilon i$ for $x \leq 0$ until they cut the circle $|z+1|=r$, which close the contour at the points $-1-r(\varepsilon) \pm \varepsilon i$, where $0<r(\varepsilon) \rightarrow r$ as $\varepsilon \rightarrow 0$. By the formula (11), we have

$$
\begin{aligned}
F\left(z_{0}\right)= & \frac{1}{2 \pi i}\left[\int_{\pi / 2}^{-\pi / 2} \frac{i \varepsilon e^{\theta i} F\left(\varepsilon e^{\theta i}-1\right)}{\varepsilon e^{\theta i}-1-z_{0}} \mathrm{~d} \theta+\int_{-r(\varepsilon)}^{0} \frac{F(x-1+\varepsilon i)}{x-1+\varepsilon i-z_{0}} \mathrm{~d} x\right. \\
& \left.+\int_{0}^{-r(\varepsilon)} \frac{F(x-1-\varepsilon i)}{x-1-\varepsilon i-z_{0}} \mathrm{~d} x+\int_{\arg [-r(\varepsilon)-\varepsilon i]}^{\arg [-r(\varepsilon)+\varepsilon i]} \frac{i r e^{\theta i} F\left(r e^{\theta i}-1\right)}{r e^{\theta i}-1-z_{0}} \mathrm{~d} \theta\right] .
\end{aligned}
$$

By the formula (8), it follows that

$$
\lim _{\varepsilon \rightarrow 0^{+}} \int_{\pi / 2}^{-\pi / 2} \frac{i \varepsilon e^{\theta i} F\left(\varepsilon e^{\theta i}-1\right)}{\varepsilon e^{\theta i}-1-z_{0}} \mathrm{~d} \theta=0 .
$$

In virtue of the limit (9), it can be derived that

$$
\begin{aligned}
& \lim _{\substack{\varepsilon \rightarrow 0^{+} \\
r \rightarrow \infty}} \int_{\arg [-r(\varepsilon)-\varepsilon i]}^{\arg [-r(\varepsilon)+\varepsilon i]} \frac{i r e^{\theta i} F\left(r e^{\theta i}-1\right)}{r e^{\theta i}-1-z_{0}} \mathrm{~d} \theta \\
= & \lim _{r \rightarrow \infty} \int_{-\pi}^{\pi} \frac{i r e^{\theta i} F\left(r e^{\theta i}-1\right)}{r e^{\theta i}-1-z_{0}} \mathrm{~d} \theta \\
= & 0 .
\end{aligned}
$$


Making use of the obvious fact that $F(\bar{z})=\overline{F(z)}$ and the limit (10) yields that

$$
\begin{aligned}
& \int_{-r(\varepsilon)}^{0} \frac{F(x-1+\varepsilon i)}{x-1+\varepsilon i-z_{0}} \mathrm{~d} x+\int_{0}^{-r(\varepsilon)} \frac{F(x-1-\varepsilon i)}{x-1-\varepsilon i-z_{0}} \mathrm{~d} x \\
= & \int_{-r(\varepsilon)}^{0}\left[\frac{F(x-1+\varepsilon i)}{x-1+\varepsilon i-z_{0}}-\frac{F(x-1-\varepsilon i)}{x-1-\varepsilon i-z_{0}}\right] \mathrm{d} x \\
= & 2 i \int_{-r(\varepsilon)}^{0} \frac{\left(x-1-z_{0}\right) \Im F(x-1+\varepsilon i)-\varepsilon \Re F(x-1+\varepsilon i)}{\left(x-1+\varepsilon i-z_{0}\right)\left(x-1-\varepsilon i-z_{0}\right)} \mathrm{d} x \\
\rightarrow & 2 i \int_{-r}^{0} \frac{\lim _{\varepsilon \rightarrow 0^{+}} \Im F(x-1+\varepsilon i)}{x-1-z_{0}} \mathrm{~d} x \\
= & -2 i \int_{0}^{r} \frac{\lim _{\varepsilon \rightarrow 0^{+}} \Im F(-t-1+\varepsilon i)}{t+1+z_{0}} \mathrm{~d} t \\
\rightarrow & -2 i \int_{0}^{\infty} \frac{\lim _{\varepsilon \rightarrow 0^{+}} \Im F(-t-1+\varepsilon i)}{t+1+z_{0}} \mathrm{~d} t \\
= & 2 \pi i \int_{0}^{\infty} \frac{t+1}{t\left[(\ln t)^{2}+\pi^{2}\right]} \frac{\mathrm{d} t}{t+1+z_{0}}
\end{aligned}
$$

as $\varepsilon \rightarrow 0^{+}$and $r \rightarrow \infty$. Substituting equations (13), (14), and (15) into (12) and simplifying produce the integral representation (7). The proof of Lemma 2 is complete.

Second proof of Lemma 2. In all treatments of Pick functions, a main example is the principal logarithm ln defined in the cut plane $\mathcal{A}$ as well as

$$
-\frac{1}{\ln z}=-\frac{1}{z-1}+\int_{-\infty}^{0} \frac{1}{(t-z)\left[(\ln t)^{2}+\pi^{2}\right]} \mathrm{d} t
$$

This formula is equivalent to $[2,(1.4)]$. Multiplying the identity

$$
\int_{0}^{\infty} \frac{1}{t\left[(\ln t)^{2}+\pi^{2}\right]}=1
$$

by $\frac{1}{z}$ and inserting it in the previous formula yield

$$
\frac{z-1}{z \ln z}=\int_{0}^{\infty}\left[\frac{1}{t z}+\frac{z-1}{z(t+z)}\right] \frac{\mathrm{d} t}{(\ln t)^{2}+\pi^{2}}=\int_{0}^{\infty} \frac{1+t}{(t+z)\left[(\ln t)^{2}+\pi^{2}\right]} \mathrm{d} t,
$$

which is the formula (7). The proof of Lemma 2 is complete.

\section{Proofs of theorems}

Now we prove Theorems 1 to 4 and Corollary 1 .

First proof of Theorem 1. By (6), we have

$$
\frac{x}{\ln (1+x)}=1+\int_{1}^{\infty} \frac{1}{[\ln (t-1)]^{2}+\pi^{2}} \frac{x}{x+t} \mathrm{~d} t
$$


and

$$
\begin{aligned}
{\left[\frac{x}{\ln (1+x)}\right]^{(k)} } & =\int_{1}^{\infty} \frac{1}{[\ln (t-1)]^{2}+\pi^{2}}\left(\frac{x}{x+t}\right)^{(k)} \mathrm{d} t \\
& =\int_{1}^{\infty} \frac{1}{[\ln (t-1)]^{2}+\pi^{2}}\left(1-\frac{t}{x+t}\right)^{(k)} \mathrm{d} t \\
& =(-1)^{k+1} k ! \int_{1}^{\infty} \frac{t}{[\ln (t-1)]^{2}+\pi^{2}} \frac{1}{(x+t)^{k+1}} \mathrm{~d} t
\end{aligned}
$$

for $k \in \mathbb{N}$. On the other hand, by (1), we also have

$$
\left[\frac{x}{\ln (1+x)}\right]^{(k)}=\sum_{n=k}^{\infty} b_{n} \frac{n !}{(n-k) !} x^{n-k}
$$

Combining (17) with (18) leads to

$$
\sum_{n=k}^{\infty} b_{n} \frac{n !}{(n-k) !} x^{n-k}=(-1)^{k+1} k ! \int_{1}^{\infty} \frac{t}{[\ln (t-1)]^{2}+\pi^{2}} \frac{1}{(x+t)^{k+1}} \mathrm{~d} t .
$$

Letting $x \rightarrow 0^{+}$on both sides of the above equation produces

$$
k ! b_{k}=(-1)^{k+1} k ! \int_{1}^{\infty} \frac{1}{[\ln (t-1)]^{2}+\pi^{2}} \frac{1}{t^{k}} \mathrm{~d} t .
$$

Thus, the formula (2) is proved.

Second proof of Theorem 1. By the integral representation (7), we have

$$
\frac{x}{\ln (1+x)}=\int_{1}^{\infty} \frac{t}{(t-1)\left\{[\ln (t-1)]^{2}+\pi^{2}\right\}} \frac{1+x}{x+t} \mathrm{~d} t
$$

and

$$
\begin{aligned}
{\left[\frac{x}{\ln (1+x)}\right]^{(k)} } & =\int_{1}^{\infty} \frac{t}{(t-1)\left\{[\ln (t-1)]^{2}+\pi^{2}\right\}}\left(\frac{1+x}{x+t}\right)^{(k)} \mathrm{d} t \\
& =\int_{1}^{\infty} \frac{t}{(t-1)\left\{[\ln (t-1)]^{2}+\pi^{2}\right\}}\left(1+\frac{1-t}{x+t}\right)^{(k)} \mathrm{d} t \\
& =(-1)^{k+1} k ! \int_{1}^{\infty} \frac{t}{[\ln (t-1)]^{2}+\pi^{2}} \frac{1}{(x+t)^{k+1}} \mathrm{~d} t
\end{aligned}
$$

for $k \in \mathbb{N}$. Combining (19) with (18) leads to

(20) $\sum_{n=k}^{\infty} b_{n} \frac{n !}{(n-k) !} x^{n-k}=(-1)^{k+1} k ! \int_{1}^{\infty} \frac{t}{[\ln (t-1)]^{2}+\pi^{2}} \frac{1}{(x+t)^{k+1}} \mathrm{~d} t$.

Letting $x \rightarrow 0^{+}$on both sides of (20) yields the formula (2). The proof of Theorem 1 is complete. 
First proof of Theorem 2. Theorem 4a in [21, p. 108] reads that a necessary and sufficient condition that the sequence $\left\{\mu_{n}\right\}_{0}^{\infty}$ should have the expression

$$
\mu_{n}=\int_{0}^{1} t^{n} \mathrm{~d} \alpha(t)
$$

for $n \geq 0$, where $\alpha(t)$ is non-decreasing and bounded for $0 \leq t \leq 1$, is that it should be completely monotonic. Theorem 14a in [21, p. 164] states that a completely monotonic sequence $\left\{\mu_{n}\right\}_{n \geq 0}$ is minimal if and only if the equality (21) is valid for $n \geq 0$ and $\alpha(t)$ is a non-decreasing bounded function continuous at $t=0$.

Setting in the equality (21)

$$
\alpha(t)=\int_{0}^{t} \frac{1}{s\left\{[\ln (1 / s-1)]^{2}+\pi^{2}\right\}} \mathrm{d} s
$$

for $t \in[0,1]$ and $\alpha(1)=b_{1}=\frac{1}{2}$ yields the required complete monotonicity and minimality.

Second proof of Theorem 2. From (2), it follows that for $n \in \mathbb{N}$

$$
\begin{aligned}
(-1)^{n+1} b_{n} & =\int_{1}^{\infty} \frac{1}{\left\{[\ln (t-1)]^{2}+\pi^{2}\right\} t^{n}} \mathrm{~d} t \\
& =\int_{1}^{0} \frac{1}{\left\{[\ln (1 / s-1)]^{2}+\pi^{2}\right\}} s^{n} \mathrm{~d}\left(\frac{1}{s}\right) \\
& =\int_{0}^{1} \frac{1}{\left\{[\ln (1 / s-1)]^{2}+\pi^{2}\right\}} s^{n-2} \mathrm{~d} s \\
& =\int_{0}^{1} \frac{1}{s\left\{[\ln (1 / s-1)]^{2}+\pi^{2}\right\}} s^{n-1} \mathrm{~d} s \\
& \triangleq c_{n-1} .
\end{aligned}
$$

Since $c_{0}=b_{1}=\frac{1}{2}$ and the function $\frac{1}{s\left\{[\ln (1 / s-1)]^{2}+\pi^{2}\right\}}$ is positive on $(0,1)$, then the complete monotonicity and minimality of the sequence $\left\{c_{n}\right\}_{0}^{\infty}$ is readily obtained. The proof of Theorem 2 is complete.

Proof of Theorem 3. A function $f$ is said to be completely monotonic on an interval $I$ if $f$ has derivatives of all orders on $I$ and $(-1)^{n} f^{(n)}(x) \geq 0$ for $x \in I$ and $n \geq 0$. See [11, Chapter XIII] and [21, Chapter IV].

From the proofs of Theorem 1, we observe that

$$
b_{n}=(-1)^{n+1} \lim _{x \rightarrow 0^{+}} h_{n}(x)
$$

and

$$
h_{n}(x)=\int_{1}^{\infty} \frac{1}{\left\{[\ln (t-1)]^{2}+\pi^{2}\right\}(t+x)^{n}} \mathrm{~d} t
$$

is completely monotonic on $[0, \infty)$. 
In [10], or see [11, p. 367], it was obtained that if $f$ is a completely monotonic function on $[0, \infty)$, then

$$
\left|f^{\left(a_{i}+a_{j}\right)}(x)\right|_{m} \geq 0
$$

and

$$
\left|(-1)^{a_{i}+a_{j}} f^{\left(a_{i}+a_{j}\right)}(x)\right|_{m} \geq 0
$$

where $\left|a_{i j}\right|_{m}$ denotes a determinant of order $m$ with elements $a_{i j}$ and $a_{i}$ for $1 \leq i \leq m$ are nonnegative integers. Applying $f$ in (24) and (25) to the function $h_{n}(x)$ yields

and

$$
\left|h_{n}^{\left(a_{i}+a_{j}\right)}(x)\right|_{m} \geq 0
$$

$$
\left|(-1)^{a_{i}+a_{j}} h_{n}^{\left(a_{i}+a_{j}\right)}(x)\right|_{m} \geq 0
$$

that is,

$$
\left|(-1)^{a_{i}+a_{j}} \frac{\left(n+a_{i}+a_{j}-1\right) !}{(n-1) !} h_{n+a_{i}+a_{j}}(x)\right|_{m} \geq 0
$$

and

$$
\left|\frac{\left(n+a_{i}+a_{j}-1\right) !}{(n-1) !} h_{n+a_{i}+a_{j}}(x)\right|_{m} \geq 0 .
$$

Letting $x \rightarrow 0^{+}$in (26) and (27) and making use of (22) produce

$$
\left|(-1)^{a_{i}+a_{j}} \frac{\left(n+a_{i}+a_{j}-1\right) !}{(n-1) !}(-1)^{n+a_{i}+a_{j}+1} b_{n+a_{i}+a_{j}}\right|_{m} \geq 0
$$

and

$$
\left|\frac{\left(n+a_{i}+a_{j}-1\right) !}{(n-1) !}(-1)^{n+a_{i}+a_{j}+1} b_{n+a_{i}+a_{j}}\right|_{m} \geq 0 .
$$

Further simplifying (28) and (29) leads to

$$
\left|(-1)^{n+1}\left(n+a_{i}+a_{j}-1\right) ! b_{n+a_{i}+a_{j}}\right|_{m} \geq 0
$$

and

$$
\left|(-1)^{n+a_{i}+a_{j}+1}\left(n+a_{i}+a_{j}-1\right) ! b_{n+a_{i}+a_{j}}\right|_{m} \geq 0,
$$

which are equivalent to (3) and (4). Theorem 3 is thus proved.

Proof of Theorem 4. In [20, p. 106, Theorem A] and [11, p. 367, Theorem 2], a minor correction of $[6$, Theorem 1$]$, it was obtained that if $f$ is a completely monotonic function on $(0, \infty)$ and $\lambda \preceq \mu$, then

$$
\left|\prod_{i=1}^{n} f^{\left(\lambda_{i}\right)}(x)\right| \leq\left|\prod_{i=1}^{n} f^{\left(\mu_{i}\right)}(x)\right| \text {. }
$$

Applying the inequality (30) to $h_{n}(x)$, defined by (23), creates

$$
\left|\prod_{i=1}^{m}(-1)^{\lambda_{i}} \frac{\left(n+\lambda_{i}-1\right) !}{(n-1) !} h_{n+\lambda_{i}}(x)\right| \leq\left|\prod_{i=1}^{m}(-1)^{\mu_{i}} \frac{\left(n+\mu_{i}-1\right) !}{(n-1) !} h_{n+\mu_{i}}(x)\right|
$$


which can be simplified as

$$
\left|\prod_{i=1}^{m}\left(n+\lambda_{i}-1\right) ! h_{n+\lambda_{i}}(x)\right| \leq\left|\prod_{i=1}^{m}\left(n+\mu_{i}-1\right) ! h_{n+\mu_{i}}(x)\right| .
$$

Further taking $x \rightarrow 0^{+}$and utilizing (22) turn out

$$
\left|\prod_{i=1}^{m}\left(n+\lambda_{i}-1\right) !(-1)^{n+\lambda_{i}+1} b_{n+\lambda_{i}}\right| \leq\left|\prod_{i=1}^{m}\left(n+\mu_{i}-1\right) !(-1)^{n+\mu_{i}+1} b_{n+\mu_{i}}\right|
$$

which is equivalent to (5). The proof of Theorem 4 is complete.

Proof of Corollary 1. It is clear that $(i, i+2) \succ(i+1, i+1)$ for $i \geq 0$. Therefore, by virtue of (5), we have

$$
\left(i ! b_{i+1}\right)\left[(i+2) ! b_{i+3}\right] \geq\left[(i+1) ! b_{i+2}\right]^{2} .
$$

This implies the required logarithmic convexity.

This conclusion can also be deduced from Theorem 3. The proof of Theorem 1 is thus complete.

\section{Remarks}

Finally, we would like to give some remarks on something related to the integral representations (6) and (7).

Remark 1. In [1, p. 230, 5.1.32], it is listed that

As a result, we have

$$
\ln \frac{b}{a}=\int_{0}^{\infty} \frac{e^{-a u}-e^{-b u}}{u} \mathrm{~d} u .
$$

$$
\ln [\ln (1+x)]=\int_{0}^{\infty} \frac{e^{-u}-e^{-u \ln (1+x)}}{u} \mathrm{~d} u=\int_{0}^{\infty} \frac{e^{-u}-(1+x)^{-u}}{u} \mathrm{~d} u
$$

and, by a differentiation,

$$
\begin{aligned}
\frac{1}{(1+x) \ln (1+x)} & =\int_{0}^{\infty} \frac{1}{(1+x)^{u+1}} \mathrm{~d} u \\
& =\int_{0}^{\infty}\left[\frac{1}{\Gamma(1+u)} \int_{0}^{\infty} t^{u} e^{-(1+x) t} \mathrm{~d} t\right] \mathrm{d} u \\
& =\int_{0}^{\infty}\left[\int_{0}^{\infty} \frac{t^{u}}{\Gamma(1+u)} \mathrm{d} u\right] e^{-(1+x) t} \mathrm{~d} t
\end{aligned}
$$

where $\Gamma(z)$ is the classical gamma function which may be defined by the Euler integral

$$
\Gamma(z)=\int_{0}^{\infty} t^{z-1} e^{-t} \mathrm{~d} t, \quad \Re(z)>0 .
$$

The integral representation (31) means that $\frac{1}{(1+x) \ln (1+x)}$ is a completely monotonic function on $(0, \infty)$. In other words, the function $\frac{1}{\ln (1+x)}$ is logarithmically 
completely monotonic on $(0, \infty)$. More strongly, it was claimed in [3, p. 2130, $(34)]$ and $\left[4\right.$, p. 12, (33)] that the function $\frac{1}{\ln (1+x)}$ is a Stieltjes transform. For information on the notions "logarithmically completely monotonic function" and "Stieltjes transform", please refer to [14, Remark 8], [15, Section 1], [16, Remark 4.7], the monograph [18], and many other closely-related references therein.

From (31) and by integration by part, it is not difficult to obtain that

$$
\frac{1}{\ln (1+x)}=\int_{0}^{\infty}\left[\int_{0}^{\infty} \frac{t^{u-1}}{\Gamma(u)} \mathrm{d} u\right] e^{-(1+x) t} \mathrm{~d} t, \quad x>0 .
$$

By induction and integration by part, we can obtain

$$
\begin{aligned}
\frac{(1+x)^{k}}{\ln (1+x)} & =\int_{0}^{\infty}\left[\int_{0}^{\infty} \frac{t^{u-k-1}}{\Gamma(u-k)} \mathrm{d} u\right] e^{-(1+x) t} \mathrm{~d} t \\
& =\int_{0}^{\infty}\left[\int_{-k}^{\infty} \frac{t^{u-1}}{\Gamma(u)} \mathrm{d} u\right] e^{-(1+x) t} \mathrm{~d} t
\end{aligned}
$$

for $x>0$ and $k \in \mathbb{Z}$, where $\mathbb{Z}$ denotes the set of all integers and the classical gamma function $\Gamma(z)$ given in (32) may be extended analytically to $\mathbb{C} \backslash\{0,-1,-2, \ldots\}$ by the Gauss formula

$$
\Gamma(z)=\lim _{n \rightarrow \infty} \frac{n ! n^{z}}{z(z+1) \cdots(z+n)} .
$$

See [19, Section 1.1].

Remark 2. By the way, the term $\frac{1}{z}$ in (6) was lost in $[3$, p. $2130,(34)]$ and $[4$, p. $12,(33)]$ and was corrected in $[17,22]$.

Remark 3. The integral representation (7) in Lemma 2 has been utilized in the paper [13].

Remark 4. A function $f: I \subseteq(0, \infty) \rightarrow[0, \infty)$ is called a Bernstein function on $I$ if $f(x)$ has derivatives of all orders and $f^{\prime}(x)$ is completely monotonic on $I$. See the monograph [18]. We claim that the generating function $\frac{x}{\ln (1+x)}$ of the Bernoulli numbers of the second kind $b_{k}$ is a Bernstein function on $(0, \infty)$. This can be proved by two approaches below.

(1) The integral representation (16) shows us that the function $\frac{x}{\ln (1+x)}$ is positive and increasing on $(0, \infty)$. The integral representation (17) reveals that the first derivative of $\frac{x}{\ln (1+x)}$ is completely monotonic on $(0, \infty)$. So the function $\frac{x}{\ln (1+x)}$ is a Bernstein function on $(0, \infty)$.

(2) It is not difficult to see that

$$
\frac{x}{\ln (1+x)}=\int_{0}^{1}(1+x)^{t} \mathrm{~d} t
$$

and the function $(1+x)^{t}$ for $t \in(0,1)$ is a Bernstein function. 
Remark 5 . This paper is a combined and revised version of the preprints $[12,17]$ and Chapter 5 of the thesis [22].

Acknowledgements. The authors appreciate anonymous referees for their careful corrections to and valuable comments on the original version of this paper.

\section{References}

[1] M. Abramowitz and I. A. Stegun (Eds), Handbook of Mathematical Functions with Formulas, Graphs, and Mathematical Tables, National Bureau of Standards, Applied Mathematics Series 55, 10th printing, Washington, 1972.

[2] C. Berg, A Pick function related to an inequality for the entropy function, J. Inequal. Pure Appl. Math. 2 (2001), no. 2, Art. 26, 3 pp.

[3] C. Berg and H. L. Pedersen, A one-parameter family of Pick functions defined by the Gamma function and related to the volume of the unit ball in $n$-space, Proc. Amer. Math. Soc. 139 (2011), no. 6, 2121-2132.

[4] - A Pick function related to the sequence of volumes of the unit ball in $n$-space, available online at http://arxiv.org/abs/0912.2185.

[5] L. Comtet, Advanced Combinatorics: The Art of Finite and Infinite Expansions, Revised and Enlarged Edition, D. Reidel Publishing Co., Dordrecht and Boston, 1974.

[6] A. M. Fink, Kolmogorov-Landau inequalities for monotone functions, J. Math. Anal. Appl. 90 (1982), no. 1, 251-258.

[7] T. W. Gamelin, Complex Analysis, Undergraduate Texts in Mathematics, Springer, New York-Berlin-Heidelberg, 2001.

[8] G. H. Hardy, J. E. Littlewood, and G. Pólya, Inequalities, Cambridge University Press, Cambridge, 1934.

[9] A. W. Marshall, I. Olkin, and B. C. Arnold, Inequalities: Theory of Majorization and its Applications, 2nd Ed., Springer Verlag, New York-Dordrecht-Heidelberg-London, 2011.

[10] D. S. Mitrinović and J. E. Pečarić, On two-place completely monotonic functions, Anzeiger Öster. Akad. Wiss. Math.-Natturwiss. Kl. 126 (1989), 85-88.

[11] D. S. Mitrinović, J. E. Pečarić, and A. M. Fink, Classical and New Inequalities in Analysis, Kluwer Academic Publishers, Dordrecht-Boston-London, 1993.

[12] F. Qi, An integral representation and properties of Bernoulli numbers of the second kind, available online at http://arxiv.org/abs/1301.7181.

[13] _ An integral representation, complete monotonicity, and inequalities of Cauchy numbers of the second kind, J. Number Theory 144 (2014), 244-255.

[14] F. Qi, S. Guo, and B.-N. Guo, Complete monotonicity of some functions involving polygamma functions, J. Comput. Appl. Math. 233 (2010), no. 9, 2149-2160.

[15] F. Qi, Q.-M. Luo, and B.-N. Guo, Complete monotonicity of a function involving the divided difference of digamma functions, Sci. China Math. 56 (2013), no. 11, 2315-2325.

[16] F. Qi, C.-F. Wei, and B.-N. Guo, Complete monotonicity of a function involving the ratio of gamma functions and applications, Banach J. Math. Anal. 6 (2012), no. 1, $35-44$.

[17] F. Qi and X.-J. Zhang, A Stieltjes function involving the logarithmic function and an application, available online at http://arxiv.org/abs/1301.6425.

[18] R. L. Schilling, R. Song, and Z. Vondraček, Bernstein Functions-Theory and Applications, 2nd ed., de Gruyter Studies in Mathematics 37, Walter de Gruyter, Berlin, Germany, 2012

[19] H. M. Srivastava and J. Choi, Zeta and q-Zeta Functions and Associated Series and Integrals, Elsevier Science Publishers, Armsterdam-London-New York, 2012. 
[20] H. van Haeringen, Completely monotonic and related functions, J. Math. Anal. Appl. 204 (1996), no. 2, 389-408.

[21] D. V. Widder, The Laplace Transform, Princeton University Press, Princeton, 1946.

[22] X.-J. Zhang, Integral Representations, Properties, and Applications of Three Classes of Functions, Thesis supervised by Professor Feng Qi and submitted for the Master Degree of Science in Mathematics at Tianjin Polytechnic University in January 2013.

FEng QI

School of Mathematics and Informatics

Henan Polytechnic University

Jiaozuo City, Henan Province, 454010, P. R. China

AND

College of Mathematics

InNer Mongolia University FOR NATIONALities

Tongliao City, Inner Mongolia Autonomous Region, 028043, P. R. China

AND

Department of Mathematics

SCHOOL OF SCIENCE

Tianjin Polytechnic University

Tianjin City, 300387, P. R. China

E-mail address: qifeng618@gmail.com, qifeng618@hotmail.com, qifeng618@qq.com

XIAO-JING ZHANG

The 59Th Middle SCHOOL

Jianxi District, Luoyang City

Henan Province, 471000, P. R. China

AND

Department of Mathematics

SCHOOL OF SCIENCE

Tianjin Polytechnic University

Tianjin City, 300387, P. R. China

E-mail address: xiao.jing.zhang@qq.com 